PROCEEDINGS OF THE

AMERICAN MATHEMATICAL SOCIETY

Volume 129, Number 7, Pages 1899-1906

S 0002-9939(00)05865-2

Article electronically published on December 4, 2000

\title{
ON THE SLOPE OF BIELLIPTIC FIBRATIONS
}

\author{
MIGUEL A. BARJA \\ (Communicated by Ron Donagi) \\ A la memoria de Fernando
}

\begin{abstract}
Let $\pi: S \longrightarrow B$ be a bielliptic fibration. We prove $S$ is, up to base change, a rational double cover of an elliptic fibration and that $\pi$ is isotrivial provided it is smooth. Finally, we prove that the slope of $\pi$ is at least four provided the genus of the fibre is at least six.
\end{abstract}

\section{IntRoduCtion}

Let $\pi: S \longrightarrow B$ be a fibration, i.e. a surjective morphism with connected fibres, from a smooth projective surface $S$ onto a smooth curve $B$. A fibration is said to be relatively minimal when it has no vertical $(-1)$-curve. Let $g$ denote the genus of a general fibre and $b$ the genus of $B$.

Let $\omega_{S / B}=\omega_{S} \otimes \pi^{*}\left(\omega_{B}^{-1}\right)$ be the relative canonical bundle and let $\Delta(\pi):=$ $\operatorname{deg} \pi_{*}\left(\omega_{S / B}\right)$. It is known that $\Delta(\pi) \geq 0$ and that $\Delta(\pi)=0$ if and only if $\pi$ is locally trivial with respect to the Zariski topology (see [3], III, theorem 18.2). Assume $\pi$ is not locally trivial. Then we define the slope of $\pi$ as

$$
\lambda(\pi):=\omega_{S / B}^{2} / \Delta(\pi)
$$

(see [19]). There are several results on the lower slope of relatively minimal fibrations of genus $g \geq 2$. First of all we have $\lambda \geq 4-\frac{4}{g}$ (see [8], [12, [13, 18] when the general fibre is hyperelliptic and [19] for the general case) and equality holds only in the hyperelliptic case ([9]). There are improvements in the non-hyperelliptic case for $g \leq 5$ (see [4], [7], [9], [11, [14]) but the presently-known techniques seem to have some limitations to extend these results to higher genus.

Recently Konno has being trying to find good bounds depending on some extra numerical invariants of the general fibre, such as the Clifford index. In [10, Konno finds better bounds for trigonal and plane quintic fibrations (so Clifford index 1), although they do not seem to be sharp. Also in [11] he gets general bounds depending on the Clifford index in some cases.

In this paper we deal with the case of bielliptic fibrations (i.e., when the general fibre has a 2-to-1 map onto an elliptic curve). Using the glueing results of [2] we know that if $g \geq 6$, a bielliptic fibration is a (generically) double cover of an elliptic

Received by the editors December 12, 1997 and, in revised form, October 29, 1999.

2000 Mathematics Subject Classification. Primary 14H10; Secondary 14J29.

Partially supported by CICYT PS93-0790 and HCM project n.ERBCHRXCT-940557. 
fibration. We prove (Example 1.2) that this is not true in general if $g \leq 5$ due to the existence of several bielliptic maps in the general fibre.

Using this we get the following sharp bound for the slope of bielliptic fibrations.

Theorem 2.1. Let $\pi: S \longrightarrow B$ be a relatively minimal bielliptic fibration of genus $g \geq 6$. Let $V$ be the relative minimal model of the elliptic fibration obtained in section 1 . Then

(a) $\lambda(\pi) \geq 4+\frac{2(g-5) \chi \mathcal{O}_{V}}{\Delta(\pi)} \geq 4$.

(b) $\lambda(\pi)=4$ if and only if $S$ is the minimal desingularization of a double cover of a smooth elliptic surface $V$ such that:

- All the fibres of the elliptic fibration $\tau: V \longrightarrow B$ are smooth and isomorphic.

- The branch divisor of the double cover has only negligible singularities.

In particular, the bound is sharp.

The author thanks, among others, Professor Juan Carlos Naranjo for his encouragement and interesting comments.

During the final revision of this paper the advisor of the author, Professor Fernando Serrano, passed away. The author would like to thank him heartfully for his support and continuous help, not only during the preparation of this work but also during the last years for his teaching and friendship.

Throughout this paper we work over the field of complex numbers $\mathbb{C}$.

\section{Bielliptic Fibrations}

Let $F$ be a smooth curve of genus $g$. The curve $F$ is bielliptic if $F$ admits a 2-to-1 map onto an elliptic smooth curve $E$. Such a map is always given by the quotient by an involution $\mathfrak{i} \in \operatorname{Aut}(F)$, called a bielliptic involution on $F$. It is a well-known fact that such an involution is unique if $g \geq 6$ (see for example [1, page 280, exercise I-9).

Let $\pi: S \longrightarrow B$ be a fibration of genus $g$. We say that $\pi$ is bielliptic if the general fibre $F$ of $\pi$ is also. The following result clarifies the structure of such fibrations. The fibration $\pi$ is said to be smooth if every fibre is smooth and it is said to be isotrivial if all the smooth fibres are mutually isomorphic.

Proposition 1.1. Let $\pi: S \longrightarrow B$ be a bielliptic fibration of genus $g$. Then:

(a) A base change of $S$ is a rational double cover of an elliptic surface over the base curve.

(b) If $g \geq 6$ the same is true without base change.

(c) If $\pi$ is smooth, then $\pi$ is isotrivial.

Proof. (a) and (b) are consequences of general results given in 2. We give here a sketch of the proof and refer there for details.

Given $\pi: S \longrightarrow B$ we can consider $\psi:{\underline{\text { uut }_{S}}}_{S / B}^{2,2 g-2} \longrightarrow B$ the scheme of relative automorphisms of $S$ over $B$ of order 2 having $2 g-2$ fixed points (which corresponds fibrewise to double covers of elliptic curves) which is a quasi-projective $B$-scheme. After a base change $B^{\prime} \longrightarrow B$ such a map always has a section defined over a nonempty Zariski open subset of $B^{\prime}$ which corresponds to a rational automorphism $\Phi$ of the minimal desingularization $S^{\prime}$ of $S \times{ }_{B} B^{\prime}$ such that $\Phi_{\mid F_{t}}$ is a bielliptic involution 
for $t \in B^{\prime}$ general. If $V$ is a desingularization of $S^{\prime} /\langle\Phi\rangle$ we have a rational double cover $S^{\prime}--\rightarrow V$ over $B^{\prime}$.

If $g \geq 6$, then $\psi$ is 1 -to- 1 and base change is not needed in order to have a section.

(c) Isotriviality can be checked after base change. Following [2], section 2, we can consider after base change

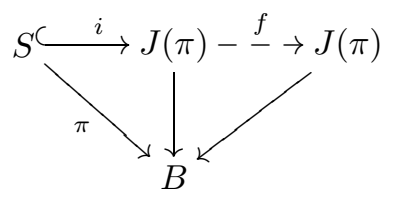

where $J(\pi)$ is the relative Jacobian variety of $S$ over $B$ and $f$ is a rational relative endomorphism of $J(\pi)$ such that $f \circ i$ produces a bielliptic map on the general fibre of $\pi$ (see [2]). Let $V=\overline{(f \circ i)(S)}$. Note that $V$ is an elliptic surface over $B$ (possibly singular). Nevertheless classification of singular fibres of a smooth elliptic surface shows, since $J(\pi)_{t}$ is an abelian variety for every $t \in B$, that $V$ is smooth and the map $\tau: V \longrightarrow B$ is also smooth. Moreover, the map $g=f \circ i: S--\rightarrow V$ can be solved after some blow-ups but then exceptional curves must be contracted since $V \subseteq J(\pi)$. So we have that $S$ is a double cover of a smooth elliptic fibration (perhaps after base change). In particular every fibre of $\pi$ is bielliptic.

Consider now the double cover $g: S \longrightarrow V$. Since $g$ has degree two the branching divisor of $g$ must be smooth and hence it is étale over $B$. If we perform base changes to the irreducible components of the branching divisor, we get a fibration for which the irreducible components of the branching divisor $D$ are sections of $\tau$. Moreover, since $\tau: V \longrightarrow B$ is a smooth elliptic fibration it is isotrivial (see [15], thms. 6,7, chapter IV) and then, after base change, we can assume $V=B \times E$ ( $E$ : elliptic smooth curve). Let $D_{1}$ be an irreducible component of $D$. If $D_{1}$ is a constant section of $\tau$, then so must be the other components and then $\pi$ is clearly isotrivial. Assume $D_{1}$ is not a constant section. Then $D_{1}=\{(b, \alpha(b)) \in B \times E \mid \alpha: B \longrightarrow$ $E$ non constant map $\}$. Fix a group structure on $E$ and consider the automorphism of $V$ over $B$ defined by $\beta(b, x)=(b, x+\alpha(b))$. Note that $\beta^{-1}\left(D_{1}\right)=B \times\{0\}$ and, hence, $\beta^{-1}(D)$ is composed of trivial horizontal sections. If we change the base

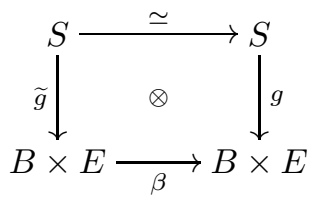

the branching divisor of $\widetilde{g}$ is just $\beta^{-1}(D)$ which is constant. Hence $\pi$ is isotrivial.

A bielliptic curve of genus $g \leq 5$ can have more than one bielliptic involution; the number of such involutions are in correspondence with the elliptic components of $W_{4}^{1}(F)$, the Brill-Noether locus of linear series on $F$ of type $g_{4}^{1}$. We give an example which shows that these involutions do not glue independently for a general fibration.

Example 1.2. We are going to give an example for which the general fibre has two different bielliptic structures which are interchanged by the monodromy. Hence, the fibration is not a double cover of an elliptic fibration. Take a genus five curve $F$ with exactly two bielliptic involutions $\sigma_{i}: F \longrightarrow E_{i}$ such that $E_{1} \nsucceq E_{2}$, with 
$E_{i}$ having no exceptional automorphisms (a count of constants shows that such an $F$ can be chosen). Then $\sigma_{1} \times \sigma_{2}: F \longrightarrow E_{1} \times E_{2}$ embeds $F$ as a smooth curve, $F \in\left|\ell_{1}^{*}\left(2 p_{1}\right) \otimes \ell_{2}^{*}\left(2 p_{2}\right)\right|$, where $\ell_{i}: E_{1} \times E_{2} \longrightarrow E_{i}$ are the projections and $\left(p_{1}, p_{2}\right) \in E_{1} \times E_{2}$. Since Aut $\left(E_{1} \times E_{2}\right)$ acts transitively on $E_{1} \times E_{2}$ we have that for every $\left(q_{1}, q_{2}\right) \in E_{1} \times E_{2}$ there exists $\widetilde{F} \in\left|\ell_{1}^{*}\left(2 q_{1}\right) \otimes \ell_{2}^{*}\left(2 q_{2}\right)\right|, \widetilde{F} \cong F$.

Let $B$ be any smooth curve having an involution $\iota$ and let $g: B \longrightarrow \bar{B}=B /\langle\iota\rangle$. Consider a morphism $\kappa: B \longrightarrow \mathbb{P}^{1}$ with no factorization through $\bar{B}$. Take a fixed $\bar{t} \in \bar{B}$ such that $g^{-1}(\bar{t})=\left\{t_{1}, t_{2}\right\}$ with $\kappa\left(t_{1}\right) \neq \kappa\left(t_{2}\right)$. After an automorphism of $\mathbb{P}^{1}$ we can suppose that $\kappa\left(t_{i}\right)$ is the modular invariant of $E_{i}$ in $\mathbb{C} \subseteq \mathbb{P}^{1}$.

Then, by [3], p. 160, there exists an elliptic fibration $\tau: V \longrightarrow B$ with a section, such that $\tau^{-1}\left(t_{i}\right) \cong E_{i}$. Let $B^{\prime}$ be the image in $V$ of the section of $\tau$. Consider the following Cartesian diagram:

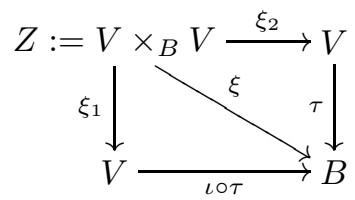

Then, for $t \in B$ we have $Z_{t}=\xi^{-1}(t)=E_{\iota(t)} \times E_{t}$, where $E_{m}=\tau^{-1}(m)$. The natural involution on $V \times_{\mathbb{C}} V$ induces commutative diagrams

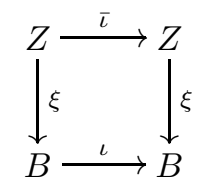

and

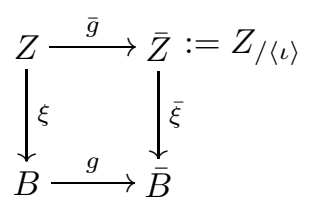

Note that $\bar{Z}$ is a threefold fibred over $\bar{B}$ and the fibre over a general $g(t) \in \bar{B}$ is $E_{\iota(t)} \times E_{t}$. We can assume $\bar{Z}$ is already smooth.

Let $B^{\prime \prime}=\bar{g}\left(\xi_{2}^{-1}\left(B^{\prime}\right)\right)$ and $\mathcal{L}=\mathcal{O}_{\bar{Z}}\left(2 B^{\prime \prime}\right)$. We have that $\mathcal{L}_{\mid \bar{Z}_{\bar{t}}} \cong \ell_{1}^{*}\left(2 q_{1}\right) \otimes \ell_{2}^{*}\left(2 q_{2}\right)$ for some $\left(q_{1}, q_{2}\right) \in E_{1} \times E_{2}$. Note that if $\mathfrak{a} \in \operatorname{Pic} \bar{B}$ is ample enough we have an epimorphism

$$
H^{0}\left(\bar{Z}, \mathcal{L} \otimes \bar{\xi}^{*}(\mathfrak{a})\right) \longrightarrow H^{0}\left(E_{1} \times E_{2}, \mathcal{L}_{\mid \bar{Z}_{\bar{t}}}\right)
$$

Since by hypothesis there exists $F \in\left|\mathcal{L}_{\mid \bar{Z}_{\bar{t}}}\right|$ we get $\bar{S} \in\left|\mathcal{L} \otimes \bar{\xi}^{*}(\mathfrak{a})\right|$, a surface fibred over $\bar{B}$, smooth at a general fibre and such that $\bar{S}_{\bar{t}}=F$. Again, we can suppose $\bar{S}$ is smooth. Let $\bar{\pi}: \bar{S} \longrightarrow \bar{B}$ and $F_{\bar{m}}=\bar{\pi}^{-1}(\bar{m})$. For $\bar{m} \in \bar{B}$ general we have that $F_{\bar{m}}$ is a smooth curve of genus 5 having at least two bielliptic involutions given by the inclusion $F_{\bar{m}} \subseteq E_{\iota(m)} \times E_{m}$ (if $g(m)=\bar{m}$ ) as a (2,2)-divisor. We claim that in general $\bar{m} \in \bar{B}, F_{\bar{m}}$ has exactly two bielliptic involutions. Since this is the case for $F=F_{\bar{t}}$ we only have to prove that having at most two of them is an open condition. Consider $W_{4}^{1}(\bar{\pi}) \longrightarrow \bar{B}$, the relative Brill-Noether locus of 
$\bar{\pi}$ (at least over an open set of $B$, see [16]), after a base change if necessary. The number of bielliptic involutions of $F_{\bar{m}}$ is given by the number of elliptic components of $W_{4}^{1}\left(F_{\bar{m}}\right) \cong W_{4}^{1}(\bar{\pi})_{\bar{m}}$. Then, having at most two of such components is obviously an open condition.

We claim that $\bar{S}$ is not a (birational) double cover of any elliptic fibration $\bar{\tau}$ : $\bar{V} \longrightarrow \bar{B}$. Indeed, assume we have a double cover $\bar{f}: \bar{S} \longrightarrow \bar{V}$ (we can suppose $\bar{f}$ everywhere defined after some blow-ups). Consider the base change diagram

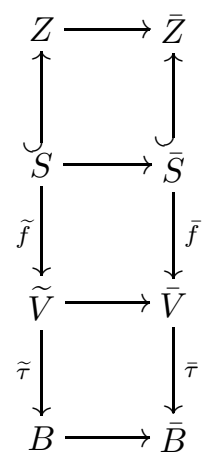

For $S$ we have three double covers of elliptic fibrations over $B$ :

$$
\begin{aligned}
& \tilde{f}: S \longrightarrow \widetilde{V}, \\
& f_{i}: S \longrightarrow V, \quad f_{i}=\xi_{i \mid S}, \quad i=1,2 .
\end{aligned}
$$

Set $U=\left\{m \in B \mid E_{m} \not E_{\iota(m)} ; E_{m}, E_{\iota(m)}\right.$ and $\widetilde{E}_{m}$ are smooth and $F_{m}$ has exactly two bielliptic involutions $\}$ (where $\widetilde{E}_{m}=\widetilde{\tau}^{-1}(m)$ ). We have that $U$ is a non-empty open set of $B$. Since $f_{1 \mid F_{m}}, f_{2 \mid F_{m}}, \widetilde{f}_{\mid F_{m}}$ are double covers of $E_{\iota(m)}, E_{m}$ and $\widetilde{E}_{m}$ respectively, we have that for every $m \in U, \widetilde{E}_{m} \cong E_{\iota(m)}$ or $\widetilde{E}_{m} \cong E_{m}$.

If $g_{1}=g \circ \iota_{\mid U}: U \longrightarrow \mathbb{P}^{1}, g_{2}=g_{\mid U}: U \longrightarrow \mathbb{P}^{1}$ and $\widetilde{g}: U \longrightarrow \mathbb{P}^{1}$ are the modular morphisms induced by $\iota \circ \tau, \tau$ and $\widetilde{\tau}$ over $U$ respectively, we have that $\widetilde{g}=g_{1}$ or $\widetilde{g}=g_{2}$. Assume $\widetilde{g}=g_{2}$.

As we have $t_{1}, t_{2} \in U$ and $\iota\left(t_{1}\right)=t_{2}$ we get

$$
E_{t_{1}}=\tau^{-1}\left(t_{1}\right)=\widetilde{\tau}^{-1}\left(t_{1}\right) \cong \widetilde{\tau}^{-1}\left(t_{2}\right)=\tau^{-1}\left(t_{2}\right)=E_{t_{2}}
$$

since $\widetilde{\tau}$ is induced by $\bar{\tau}: \bar{V} \longrightarrow \bar{B}$ and then $\widetilde{\tau}^{-1}(m) \cong \widetilde{\tau}^{-1}(\iota(m))$ for all $m \in B$. But this is imposible since by hypothesis $E_{t_{1}}=E_{1} \neq E_{2}=E_{t_{2}}$.

\section{Double COVers AND The SLOPE OF BIELliptic Fibrations}

We recall some basic facts about double covers (see [6], [3]).

By a double cover we mean a finite, degree two map between surfaces, $f_{0}: S_{0} \longrightarrow$ $V_{0}$. This map is determined by a divisor $Z_{0}$ on $V_{0}$ (the branch divisor) and a line bundle $\mathcal{L}_{0}$ such that $\mathcal{L}_{0}^{\otimes 2}=\mathcal{O}_{V_{0}}\left(Z_{0}\right)$. If $V_{0}$ is smooth, $S_{0}$ is normal (respectively smooth) if and only if $Z_{0}$ is reduced (respectively smooth).

Consider a double cover as above with $S_{0}$ normal and $V_{0}$ smooth. Then there exists a canonical resolution of singularities for $S_{0}$ which consists on a finite sequence 
of maps

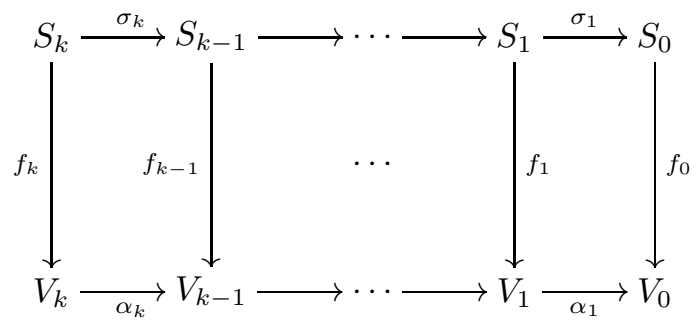

satisfying:

(i) $\alpha_{j}$ is the blow-up of $V_{j-1}$ at a singular point $p_{j-1}$ of $Z_{j-1}$ (the branching divisor of $\left.f_{j-1}\right)$.

(ii) $f_{j}$ is the double cover of $V_{j}$ defined by $\mathcal{L}_{j}^{\otimes 2} \cong \mathcal{O}\left(Z_{j}\right)$, with $Z_{j}=\alpha_{j}^{*}\left(Z_{j-1}\right)-$ $2 m_{j-1} E_{j}, \mathcal{L}_{j}=\alpha_{j}^{*}\left(\mathcal{L}_{j-1}\right) \otimes \mathcal{O}_{V_{j}}\left(-m_{j-1} E_{j}\right)$, where $E_{j}$ is the exceptional divisor of $\alpha_{j}$ and $p_{j-1}$ is a singular point of $Z_{j-1}$ of multiplicity $2 m_{j-1}$ or $2 m_{j-1}+1$.

(iii) $\sigma_{j}$ is a birational morphism induced by the cartesian product of $\alpha_{j}$ and $f_{j-1}$.

(iv) $Z_{k}$ is smooth and, hence, $S_{k}$ is a smooth surface.

Now we can use this as follows. Recall from section 1 that we have obtained $f: \widetilde{S} \longrightarrow V$ a generically 2-to-1 morphism (we can suppose that $f$ is everywhere defined up to performing blow-ups) from a blow-up of $S$ onto an elliptic fibration $V$ over $B$ which we can suppose relatively minimal after some blow-downs. Suppose that $\pi$ is relatively minimal.

Now consider

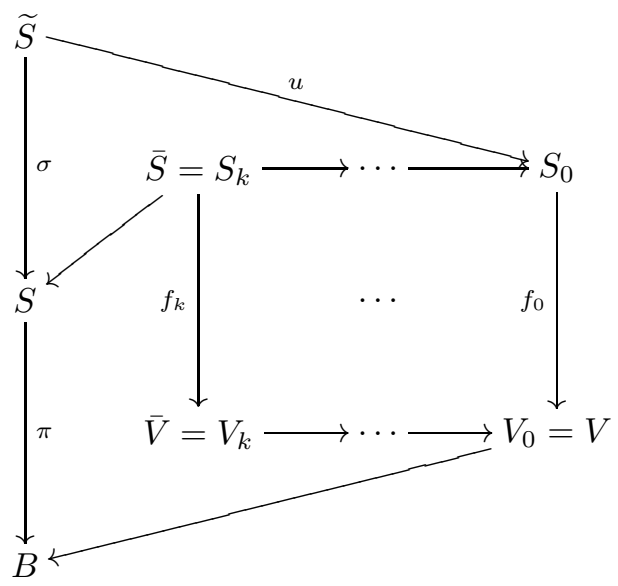

where:

- $f=f_{0} \circ u$ is the Stein factorization of $f$, with $u$ birational, $f_{0}$ finite (so it is a double cover) and $S_{0}$ normal.

- $f_{k}: S_{k} \longrightarrow V_{k}$ is the canonical resolution of singularities of $f_{0}: S_{0} \longrightarrow V_{0}$.

- $\bar{\sigma}: S_{k} \longrightarrow S$ is the birational morphism defined by the relative minimality of $\pi$.

Theorem 2.1. Let $\pi: S \longrightarrow B$ be a relatively minimal bielliptic fibration of genus $g \geq 6$. Let $V$ be the relative minimal model of the elliptic fibration obtained in section [1. Then: 
(a) $\omega_{S / B}^{2}-4 \Delta(\pi) \geq 2(g-5) \chi \mathcal{O}_{V}$. In particular, if $\pi$ is not locally trivial

$$
\lambda(\pi) \geq 4+\frac{2(g-5) \chi \mathcal{O}_{V}}{\Delta(\pi)} \geq 4
$$

(b) $\lambda(\pi)=4$ if and only if $S$ is the minimal desingularization of a double cover $S_{0} \longrightarrow V$ of a smooth elliptic surface such that:

- All the fibres of the elliptic fibration $\tau: V \longrightarrow B$ are smooth and isomorphic.

- The branch divisor of the double cover has only negligible singularities (i.e., all the multiplicities $m_{j}$ in the above process are 2 or 3 (see [13], [17])).

In particular, the bound is sharp.

Proof. (a) First of all we have

$$
\begin{aligned}
\omega_{S / B}^{2}-4 \Delta(\pi) & =\left(K_{S}^{2}-4 \chi \mathcal{O}_{S}\right)-4(b-1)(g-1) \\
& \geq\left(K_{\bar{S}}^{2}-4 \chi \mathcal{O}_{\bar{S}}\right)-4(b-1)(g-1) .
\end{aligned}
$$

For smooth double covers $f_{k}: \bar{S} \longrightarrow \bar{V}$ we have (see [3], p. 183):

$$
\begin{aligned}
\chi \mathcal{O}_{\bar{S}} & =2 \chi \mathcal{O}_{\bar{V}}+\frac{1}{2} \mathcal{L}_{k} K_{\bar{V}}+\frac{1}{2} \mathcal{L}_{k} \mathcal{L}_{k}, \\
K_{\bar{S}}^{2} & =2 K_{\bar{V}}^{2}+4 \mathcal{L}_{k} K_{\bar{V}}+2 \mathcal{L}_{k} \mathcal{L}_{k},
\end{aligned}
$$

so we have

$$
K_{\bar{S}}^{2}-4 \chi \mathcal{O}_{\bar{S}}=2\left[K_{V_{k}}^{2}-4 \chi \mathcal{O}_{V_{k}}\right]+2 \mathcal{L}_{k} K_{V_{k}} .
$$

Moreover, in each blow-up $\alpha_{j}: V_{j} \longrightarrow V_{j-1}$ we get

$$
\chi \mathcal{O}_{V_{j}}=\chi \mathcal{O}_{V_{j-1}} ; \quad K_{V_{j}}=\alpha_{j}^{*} K_{V_{j-1}}+E_{j} ; \quad \mathcal{L}_{j}=\alpha_{j}^{*} \mathcal{L}_{j-1}-m_{j-1} E_{j} .
$$

Then

$$
\begin{aligned}
& 2\left[K_{V_{j}}^{2}-4 \chi \mathcal{O}_{V_{j}}\right]+2 \mathcal{L}_{j} K_{V_{j}}=2\left[K_{V_{j-1}}^{2}-4 \chi \mathcal{O}_{V_{j-1}}\right] \\
& \quad+2 \mathcal{L}_{j-1} K_{V_{j-1}}+2\left(m_{j-1}-1\right) \geq 2\left[K_{V_{j-1}}^{2}-4 \chi \mathcal{O}_{V_{j-1}}\right]+2 \mathcal{L}_{j-1} K_{V_{j-1}} .
\end{aligned}
$$

Finally as $\tau: V \longrightarrow B$ is an elliptic minimal fibration, numerically we have $K_{V} \equiv\left[2(b-1)+\chi \mathcal{O}_{V}+\sum_{i} \frac{\left(n_{i}-1\right)}{n_{i}}\right] E([3]$, p. 162) where $E$ denotes a smooth fibre of $\tau$ and $\left\{n_{i}\right\}$ are the multiplicities of singular fibres of $\tau$. In particular $K_{V}^{2} \equiv 0$.

As $\mathcal{L}_{0}^{\otimes 2}=\mathcal{O}_{V_{0}}\left(Z_{0}\right)$ and $Z_{0}$ is the branch divisor of $f_{0}$ we get $\mathcal{L}_{0} E=(g-1)$ by Hurwitz formula. So

(4) $2\left[K_{V_{0}}^{2}-4 \chi \mathcal{O}_{V_{0}}\right]+2 \mathcal{L}_{0} K_{V_{0}}=-8 \chi \mathcal{O}_{V_{0}}$

$$
+2 \mathcal{L}_{0} E\left[2(b-1)+\chi \mathcal{O}_{V_{0}}+\sum_{i} \frac{\left(n_{i}-1\right)}{n_{i}}\right] \geq 4(b-1)(g-1)+2(g-5) \chi \mathcal{O}_{V}
$$

Then (a) follows from (1), (2), (3) and (4) and from the fact that $\chi \mathcal{O}_{V} \geq 0$ for elliptic fibrations.

(b) Looking at the proof of (a) we see that $\lambda=4$ iff $\chi \mathcal{O}_{V}=0$ and equality holds in (11), (2), (3) and (4). So we have $\lambda=4$ iff $S$ is the minimal desingularization of a double cover of an elliptic, relatively minimal, fibration $\tau: V \longrightarrow B$ such that:

- $\tau$ has no multiple fibres $\left(\forall i \quad n_{i}=1\right)$.

- $\chi \mathcal{O}_{V}=0$. 
- The branch divisor $Z_{0}$ of the double cover has only negligible singularities (see [13], [17]), i.e. all the multiplicities of the singularities of the branch divisors in the process of canonical resolution are 2 or 3 .

But the first two conditions are equivalent to the fact that $\tau$ is smooth and isotrivial (see [15], thms. 6, 7, Ch. IV). This allows us to construct examples with $\lambda(\pi)=4$ which are essentially the same as in [19], example 4.3. So the bound is sharp.

Remark 2.2. Although we cannot use double covers for the case of bielliptic fibrations of genus 5 we already know that $\lambda \geq 4$ also holds for such fibrations (see [9] thm. 5.1, 11]).

\section{REFERENCES}

[1] E. Arbarello, M. Cornalba., P.A. Griffiths, J. Harris. Geometry of algebraic curves, vol I. Grund. Math. Wiss. 267. Springer-Verlag 1985. MR 86h:14019

[2] M.A. Barja, J.C. Naranjo. Extension of maps defined on many fibres. Collect. Math. 49, 2-3 (1998), 227-238. MR 2000e:14034

[3] W. Barth, C. Peters, A. Van de Ven. Compact complex surfaces. Ergebnisse der Mathematik und ihrer Grenzgebiete 4. Springer-Verlag (1984). MR 86c:32026

[4] Z. Chen. On the bound of the slope of a non-hyperelliptic fibration of genus 4. Intern. J. Math., vol 4, No.3 (1993), 367-378.

[5] R. Hartshorne. Algebraic Geometry. Graduate Texts in Mathematics 52. Springer-Verlag (1977). MR 57:3116

[6] E. Horikawa. On deformation of Quintic Surfaces. Inventiones math. 31 (1975), 43-85.

[7] E. Horikawa. Notes on canonical surfaces. Tohoku Math. J. I 43 (1991), 141-148. MR 92e:14034

[8] E. Horikawa. Algebraic surfaces of general type with small $c_{1}^{2}$, V. J. Fac. Sci. Univ. Tokyo. Sect. A 28 (1981), 745-755. MR 84d:14019

[9] K. Konno. Non-hyperelliptic fibrations of small genus and certain irregular canonical surfaces. Ann. Sc. Norm. Pisa Ser. IV vol XX (1993), 575-595. MR 95b:14026

[10] K. Konno. A lower bound of the slope of trigonal fibrations. Intern. J. Math., vol 7, No.1 (1996), 19-27. MR 97d:14035

[11] K. Konno. Clifford index and the slope of fibred surfaces. J. Algebraic Geometry 8 (1999), 207-220. MR 2000e:14060

[12] S. Matsusaka. Some numerical invariants of hyperelliptic fibrations. J. Math. Kyoto Univ. 30-1 (1990), 33-57. MR 91a:14016

[13] U. Persson. Chern invariants of surfaces of general type. Compositio Math. 43 (1982), 3-58. MR 83b:14012

[14] M. Reid. Problems on pencils of small genus. Preprint.

[15] I.R. Shafarevich, et al. Algebraic Surfaces. Proc. Steklov Inst. Math. 75 (1965), AMS Translations, Providence R.I. (1967). MR 32:7557

[16] M. Teixidor. On translation invariance for $W_{d}^{r}$. J. reine angew. Math. 385 (1988), 10-23. MR 89a:14035

[17] G. Xiao. Surfaces fibrés en courbes de genre deux. Lect. Notes Math. 1137. Springer-Verlag 1985. MR 88a:14042

[18] G. Xiao. The fibrations of algebraic surfaces. Shangai Scientific \& Technical Publishers 1992 (Chinese).

[19] G. Xiao. Fibered algebraic surfaces with low slope. Math. Ann. 276 (1987), 449-466. MR 88a:14046

Departament de Matemàtica Aplicada I, Universitat Politècnica de Catalunya, DiAGONAl 647, 08028 Barcelona, Spain

E-mail address: barja@ma1.upc.es 